\title{
A CLASS OF GENERALIZED BEST APPROXIMATION PROBLEMS IN LOCALLY CONVEX LINEAR TOPOLOGICAL SPACES
}

\author{
HORA KRISHNA SAMANTA \\ Department of Mathematics \\ University of Burdwan \\ 713104 Burdwan (West Bengal), INDIA
}

(Received September 27, 1994 and in revised form July 15, 1996)

\begin{abstract}
In this paper a class of generalized best approximation problems is formulated in locally convex linear topological spaces and is solved, using standard results of locally convex linear topological spaces
\end{abstract}

KEY WORDS AND PHRASES: Best approximation, semi-reflexive, proximal set, continuous operators.

1991 AMS SUBJECT CLASSIFICATION CODES: 49J15, Secondary 49J20, 93C15, 93C20.

\section{INTRODUCTION}

The best approximation problem in normed linear spaces was considered by several authors including Burbu [1], Singer [2]. Also in locally convex spaces some results were obtained by Singer [3]. The principle objective of this paper is to generalize the idea of best approximation problem in locally convex linear topological space setting and to find the best control.

\section{STATEMENT OF THE PROBLEM}

Let $E$ be a convex subset of a locally convex linear topological space $X$ and $X^{*}$ be the conjugate space of all continuous linear functionals defined on $X$.

Consider the problem

$$
\min _{m \in X}\left\{\frac{1}{2} \sup _{f \in X^{\cdot}}|(m-x, f)|^{2}+I_{E}(m)\right\}
$$

where $x$ is the given element of $X$ and $I_{E}$ is the indicator function such that

$$
I_{E}(m)=\left\{\begin{array}{lll}
0 & \text { if } & m \in E \\
+\infty & \text { if } & m \notin E
\end{array} .\right.
$$

To solve this problem let us consider the following definition and theorems

DEFINITION 1. An element $l \in E$ is called a best approximation to $x \in X$ from $E$ if

$$
\sup _{f \in X^{\bullet}}|(x-l, f)| \leq \sup _{f \in X^{\cdot}}\{|(x-m, f)| \text {, for all } m \in E\} \text {. }
$$

By Remark 1.1 ([1], p 174) it can be shown that $l$ is the best approximation to $x$ from $E$, if and only if there exists $x_{0}^{*} \in X^{*}$ subject to

$$
f(l)+f^{*}\left(x_{0}^{*}\right) \leq\left(x_{0}^{*}, m\right) \text { for all } m \in E
$$


where

$$
f(m)=\frac{1}{2} \sup _{f \in X^{*}}|(m-x, f)|^{2}, m \in X
$$

THEOREM 1. An element $l \in E$ is the best approximation to $x \in X$ from elements of the convex set $E$ if and only if there exists $x_{0}^{*} \in X^{*}$ such that

(i) $\sup _{x \in X \subset X^{*}}\left|\left(x_{0}^{*}, x\right)\right|=\sup _{f \in X^{*}}|(l-x, f)|$

(ii) $\quad\left(x_{0}^{*}, m-x\right) \geq \sup _{f \in X^{*}}|(l-x, f)|^{2}, \quad$ for all $m \in E$

where $X^{* *}$ is the conjugate space of $X^{*}$.

PROOF. Now we have

$$
\begin{aligned}
f^{*}\left(x_{0}^{*}\right) & =\sup \left\{\left(x_{0}^{*}, m\right)-\frac{1}{2} \sup _{f \in X^{*}}|(m-x, f)|^{2} ; m \in X\right\} \\
& =\left(x_{0}^{*}, x\right)+\sup \left\{\left(x_{0}^{*}, m\right)-\frac{1}{2} \sup _{f \in X^{*}}|(m, f)|^{2} ; m \in X\right\} \\
& =\left(x_{0}^{*}, x\right)+\frac{1}{2} \sup _{x \in X \subset X^{* *}}\left|\left(x_{0}^{*}, x\right)\right|^{2}
\end{aligned}
$$

and the optimality condition ( 2.2 ) becomes

$$
\frac{1}{2} \sup _{f \in X^{*}}|(l-x, f)|^{2}+\frac{1}{2} \sup _{x \in X \subset X^{*}}\left|\left(x_{0}^{*}, f\right)\right|^{2} \leq\left(x_{0}^{*}, m-x\right), \forall m \in E .
$$

In particular, for $m=l$, we obtain

$$
\left(\sup _{f \in X^{*}}|(\ell-x, f)|-\sup _{x \in X \subset X^{*}}\left|\left(x_{0}^{*}, x\right)\right|\right)^{2} \leq 0
$$

which implies condition (i). Consequently from inequality (2.3) condition (ii) follows, as claimed. Conversely, it is clear that condition (i) and (ii) imply that $l$ is a best approximation, because we have

$$
\begin{aligned}
\sup _{f \in X^{*}}|(l-x, f)|^{2} & \leq\left(x_{0}^{*}, m-x\right) \\
& \leq \sup _{x \in X \subset X^{* \cdot}}\left|\left(x_{0}^{*}, x\right)\right| \cdot \sup _{f \in X^{*}}|(m-x, f)| \\
& \leq \sup _{f \in X^{*}}|(l-x, f)| \cdot \sup _{f \in X^{*}}|(m-x, f)|, \forall m \in E
\end{aligned}
$$

and so, we must have (2.1).

COROLLARY 1. If $l \in E$ is a best approximation of $x \in X$ by elements of the convex set $E$, then the following minimax relation

$$
\begin{aligned}
\sup _{f \in X^{*}}|(x-l, f)| & =\min _{m \in E} \operatorname{map}_{x \in X \subset X^{* *}} \max _{\left|\left(x^{*}, x\right)\right|=1}\left(x^{*}, m-x\right) \\
& =\max _{x \in X \subset X^{* *}} \max _{\left|\left(x^{*}, x\right)\right|=1} \min _{m \in E}\left(x^{*}, m-x\right) .
\end{aligned}
$$

holds, where $x^{*} \in X^{*}$.

PROOF. This follows clearly if we use the relationship between the solutions to the problem (P) and the existence of the saddle points $([1])$. To this end it suffices to remark that the point $x_{0}$, the 
existence of which is ensured by Theorem 1, is just the solution of the dual problem. This completes the proof. set $E$.

REMARK 1. Let $d=\sup _{f \in X^{*}}\{|(x-m, f)| ; m \in E\}$ be distance between the point $x$ and the convex

Then we obtain a weak minimax relation by replacing "min" by "inf" because in such a case only the dual problem has solutions.

Next we shall notice several special cases in which conditions (i) and (ii) of Theorem 1 have a simplified form. Namely, if $E$ is a convex cone with vertex in the origin, then condition (ii) is equivalent to the following pair of conditions

(ii') $\quad\left(x_{0}^{*}, m\right) \leq 0, \forall m \in E$, i.e. $x_{0}^{*} \in E^{0}$

(iii) $\quad\left(x_{0}^{*}, x\right)=\sup _{f \in X^{*}}|x-\ell ; f|^{2}$

where $E^{0}$ is the polar set of $E([4]$, p. 136).

Here is the argument. From condition (ii) replacing $x_{0}^{*}$ by $-x_{0}^{*}$, we obtain

$$
\left(x_{0}^{*}, x-n m\right) \geq \sup _{f \in X^{*}}|(x-l, f)|^{2}, \forall m \in E, \forall n \in N
$$

because $E$ is a cone. Therefore we cannot have $\left(x_{0}^{*}, m\right)>0$ for some element $m \in E$, that is (ii') holds.

Moreover, from properties (ii) and (ii') it follows that

$$
\begin{aligned}
\sup _{f \in X^{*}}|(x-l, f)|^{2} & \leq\left(x_{0}^{*}, x-l\right) \\
& \leq \sup _{x \in X \subset X^{* *}}\left|\left(x_{0}^{*}, x\right)\right| \sup _{f \in X^{*}}|(x-l, f)| \\
& =\sup _{f \in X^{*}}|(x-l, f)|^{2},
\end{aligned}
$$

hence $\left(x_{0}^{*}, x-l\right)=\sup _{f \in X^{*}}|(l-x, f)|^{2}$. Thus we have

$$
0 \geq\left(x_{0}^{*}, l\right)=\left(x_{0}^{*}, x\right)-\left(x_{0}^{*}, x-l\right)=\left(x_{0}^{*}, x\right)-\sup _{f \in X^{*}}|(x-l, f)|
$$

and (from (ii) if $m=0$ )

$$
\left(x_{0}^{*}, x\right) \geq \sup _{f \in X^{*}}|(x-l, f)|^{2}
$$

which implies property (ii'). The reciprocal is obvious.

When $E$ is a linear space, condition (ii') is equivalent to

$$
\left(x_{0}^{*}, m\right)=0, \forall m \in E
$$

because in this case $E=-E$.

It should be mentioned that the best approximation belongs to

$$
E \cap\left\{x \in X: \sup _{f \in X^{*}}\{|(x, f)|\} \leq d\right\}
$$

and it exists if and only if there exist separating hyperplanes which meet $E$. Moreover, the set of all best approximations is convex and coincides with the intersection of the set with any separating hyperplanes. When this intersection is non-empty the separating hyperplane is a supporting hyperplane and is given by the equation

$$
\left(x_{0}^{*}, m-x\right)=\sup _{f \in X^{*}}|(x-l, f)|^{2}, \quad m \in X .
$$


Now, let us study the existence of the best approximation. Let

$$
d=\inf _{m \in E}\left\{\sup _{f \in X^{*}}|(m-x, f)|^{2}\right\} .
$$

We easily see that

$$
\inf _{m \in E}\left\{\sup _{f \in X^{\cdot}}|(m-x, f)|\right\}=\inf _{m \in E \cap \bar{S}(x ; d+\epsilon)}\left\{\sup _{f \in X^{\cdot}}|(m-x, f)|\right\}
$$

where

$$
\bar{S}(x ; d+\epsilon)=\left\{y \in X ; \sup _{f \in X^{\cdot}}|(y-x, f)| \leq d+\epsilon\right\}, \epsilon>0 .
$$

THEOREM 2. A proper convex function $f: X \rightarrow]-\infty,+\infty]$ is a lower-semicontinuous on $X$ if and only if it is lower-semicontinuous with respect to the weak topology on $x$.

PROOF. We have already seen in Proposition 2.5 ([1], p. 12) that a convex subset of a locally convex linear topological space is (strongly) closed if and only if it is closed in the corresponding weak topology on $X$. In particular we may infer that epi $f$ is (strongly) closed if it is weakly closed. This establishes the theorem.

THEOREM 3. If the convex set $E$ is such that there exists an $\epsilon>0$ for which the set $E \cap \bar{S}(x ; d+\epsilon)$ is weakly compact, then $x$ has a best approximation in $E$.

PROOF. According to relation (2.6) it is sufficient to recall that a lower-semicontinuous function on a compact set attains its infimum. In our case, the function is obviously weakly lower-semicontinuous (see Theorem 2) on the weakly compact set $E \cap \bar{S}(m, d+\epsilon)$.

COROLLARY 2. In a semireflexive locally convex linear topological space every element possesses at least one best approximation with respect to every closed convex set.

PROOF. The set $E \cap \bar{S}(m ; d+1)$ is convex closed and bounded and hence it is weakly compact by virtue of the Alaoglu Theorem ([5], p. 15).

COROLLARY 3. In a locally convex linear topological space every element possesses at least one best approximation with respect to every closed, convex and finite dimensional set.

PROOF. In a finite dimensional space the bounded closed convex sets are compact and hence weakly compact.

DEFINITION 2. Let $X, Y$ be locally linear topological spaces of the same nature. A linear operator $T: X \rightarrow Y$ is continuous if and only if it is bounded. In other words there exists $K>0$ such that

$$
\sup _{m^{*} \in Y^{*}}\left|\left(T l, m^{*}\right)\right| \leq K \sup _{l \cdot \in X^{*}}\left|\left(l, l^{*}\right)\right|, \quad \forall l \in X .
$$

The set $L(X, Y)$ of all linear continuous operators defined on $X$ with values in $Y$ becomes a locally convex linear topological space by

$$
\begin{aligned}
\sup _{f \in L^{*}(X, Y)}|(T, f)| & =\sup \left\{\sup _{m^{*} \in Y^{*}}\left|\left(T l, m^{*}\right)\right| ; \sup _{l^{*} \in X^{*}}\left|\left(l, l^{*}\right)\right| \leq 1\right\} \\
& =\inf \left\{K ; \sup _{m^{*} \in Y^{*}}\left|\left(T l, m^{*}\right)\right| \leq K \sup _{l^{*} \in X^{*}}\left|\left(l, l^{*}\right)\right|, \quad \forall l \in X\right\} .
\end{aligned}
$$

If $Y=R$, we find that $X^{*}=L(X, R)$, called the dual of $X$, is locally convex linear topological space defined by 


$$
\sup _{l \in X \subset X^{*}}\left|\left(l^{*}, l\right)\right|=\sup \left\{\left|l^{*}(l) ; \sup _{l \cdot \in X^{*}}\right|\right\}
$$

If $X$ is real locally convex linear topological space, then

$$
\sup _{l \in X \subset X^{*}}\left|\left(l^{*}, l\right)\right|=\sup \left\{l^{*}(l) ; \sup _{l \cdot \in X^{*}}\left|\left(l, l^{*}\right)\right| \leq 1\right\} .
$$

THEOREM 4. Let $f_{0}$ be a continuous linear functional on a linear subspace $A$ of a locally convex linear topological space $X$. Then, there exists a continuous linear functional $f$ on the whole of $X$, i.e., $f \in X^{*}$, such that

(i) $\quad f / A=f_{0}$

(ii) $\sup _{l \in X \subset X^{* *}}|(f, l)|=\sup _{l \in X \subset X^{*-}}\left|\left(f_{0}, l\right)\right|$.

PROOF. Since $f_{0}$ is continuous on $A$, by relation (2.8) we have

$$
\mathrm{f}_{0}(m) \leq \sup _{l \in X \subset X^{*}}\left|\left(f_{0}, l\right)\right| \sup _{l^{*} \in X^{\bullet}}\left|\left(m, l^{*}\right)\right|, \quad \forall m \in A .
$$

By the Hahn-Banach Theorem ([1], Theorem 1.10, p. 17) for $f_{0}$ and for the convex function

$$
p(x)=\sup _{l \in X \subset X^{*}}\left|\left(f_{0}, l\right)\right| \sup _{l^{*} \in X^{*}}\left|\left(l, l^{*}\right)\right| \text {. }
$$

A specialization of this theorem yields a whole class of existence results. In this context we shall present a general and classical theorem concerning the existence of continuous linear functionals with important consequence in the duality theory of locally convex linear topological spaces.

THEOREM 5. Let $m$ be a non negative number and let $h: B \rightarrow R$ be a given real function, where $B$ is a non-empty set of the locally convex linear topological space $X$. Then, $h$ has a continuous linear extension $f$ on all of $X$ such that $\sup _{l \in X \subset X \cdot .}|(f, l)| \leq m$ if and only if the following condition holds:

$$
\left|\sum_{l=1}^{n} \lambda_{2} h\left(a_{2}\right)\right| \leq m \sup _{1^{*} \in X^{*}}\left|\left(\sum_{l=1}^{n} \lambda_{i} a_{i}, \sum_{l=1}^{n} \lambda_{2} a_{2}, l^{*}\right)\right|, \quad \forall n \in N, \lambda_{i} \in R, a_{i} \in B .
$$

PROOF. From relation (2.7a) and (2.7b) it is clear that condition (2.9) is necessary. To prove the sufficiency we consider $A=\operatorname{span} B$ and we define $f_{0}$ on $A$ by

$$
f_{0}(m)=\sum_{i=1}^{n} \lambda_{\imath} h\left(a_{\imath}\right), \quad \text { if } \quad m=\sum_{i=1}^{n} \lambda_{i} a_{\imath} \in A, a_{\imath} \in B .
$$

First, using condition (2.9) we observe that $f_{0}$ is well defined on $A$. Moreover from condition (2.9) the con-

tinuity of $f_{0}$ on $A$ follows and $\sup _{l \in X \subset X . .}\left|\left(f_{0}, l\right)\right| \leq m$. Thus any extension given under Theorem 4 has all the required properties.

THEOREM 6. For any linear subspace $A$ of a locally convex linear topological space $X$ and $l \in X$ there exists $f \in X^{*}$ with the following properties
i) $f / A=0$
ii) $f(l)=\inf _{m \in A}\left\{\sup _{l^{*} \in X^{*}}\left|\left(l-m, l^{*}\right)\right|^{2}=d(l, A)\right\}$
iii) $\sup _{l \in X \subset X^{*}}|(f, l)|=\inf _{m \in A}\left\{\sup _{l^{*} \in X^{*}}\left|\left(x-m, l^{*}\right)\right|\right\}=d(l, A)$

PROOF. We take $B=A U\{l\}$ and $h: B \rightarrow R$ defined by $h(m)=0, m \in A$, and $h(l)=d^{2}(l, A)$. We observe that for any $\lambda \neq 0$ we have 


$$
\begin{aligned}
\left|\lambda h(l)+\sum_{i=1}^{n} \lambda_{\imath} h\left(a_{\imath}\right)\right| & =|\lambda h(l)|=|\lambda| d^{2}(l, A) \leq|\lambda| d(l, A) \sup _{l^{*} \in X^{*}}\left|\left(l+\sum_{i=1}^{n} \frac{\lambda_{2}}{\lambda} a_{\imath}, l^{*}\right)\right| \\
& =d(l, A) \sup _{l \cdot \in X^{*}}\left|\left(\lambda l+\sum_{i=1}^{n} \lambda_{\imath} a_{\imath}, l^{*}\right)\right|, \quad \forall n \in N, \quad \lambda_{\imath} \in R a_{\imath} \in B
\end{aligned}
$$

which is just inequality (2.9) The desired result then follows by applying Theorem 5 Indeed, we have properties (i) and (ii) and $\sup _{l \in X \subset X . .}|(f, l)| \leq d(l, A)$. Since $m=d(l, A)$. On the other hand, if we consider a sequence $\left\{m_{n}\right\} \subset A$ such that $\sup _{l^{*} \in A}\left|\left(l+m_{n}, l^{*}\right)\right| \rightarrow d(l, A)$ we obtain

$$
\begin{aligned}
\sup _{l \in X \subset X^{*}}|(f, l)| & \geq f\left(\frac{l+m_{n}}{\sup _{l^{*} \in X^{*}}\left|\left(l+m_{n}, l^{*}\right)\right|}\right)=\frac{f(l)}{\sup _{l^{*} \in X^{*}}\left|\left(l+m_{n}, l^{*}\right)\right|} \\
& =\frac{d^{2}(l, A)}{\sup _{l^{*} \in X^{*}}\left|\left(l+m_{n}, l^{*}\right)\right|} \rightarrow d(l, A)
\end{aligned}
$$

which implies $\sup _{l \in X \subset X^{-*}}|(f, l)| \geq d(l, A)$. Hence property (iii) also holds.

COROLLARY 4. In a locally convex linear topological space $X$ for every $l \in \mathrm{X}$ there exists a continuous linear functional $f \in X^{*}$ such that

(i) $f(l)=\sup _{l^{\bullet} \in X^{*}}\left|\left(l, l^{*}\right)\right|^{2}$

(ii) $\sup _{l \in X \subset X^{* *}}|(f, l)|=\sup _{l \cdot \in X}\left|\left(l, l^{*}\right)\right|$.

Moreover, if $l \neq 0$, there exists $g \in X^{*}$ such that

(i') $g(l)=\sup _{l^{*} \in X^{*}}\left|\left(l, l^{*}\right)\right|$

(ii') $\sup _{l \in X \subset X . \cdot}|(g, l)|=l$.

PROOF. By Theorem $6, d(l, A)=\sup _{l^{*} \in X^{*}}\left|\left(l, l^{*}\right)\right|$ where $A=\{0\}$. Then the corollary completes the proof.

DEFINITION 3. The space $X$ is strictly convex if every point of the polar set

$$
\left\{l \in X: \sup _{f \in X^{-}}|(l, f)|=1\right\}
$$

is an extreme point.

THEOREM 7. A locally convex linear topological space $X$ is strictly convex if and only if the following equivalent properties hold:

(i) if $\sup _{f \in X^{*}}|(x+y, f)|=\sup _{f \in X^{\circ}}|(x, f)|+\sup _{f \in X^{*}}|(y, f)|$ and $x \neq 0$ there is $t \geq 0$ such that $y=t x$,

(ii) if $\sup _{f \in X^{\bullet}}|(x, f)|=\sup _{f \in X^{\bullet}}|(y, f)|=1$ and $x \neq y$, then $\sup _{f \in X^{\bullet}}|(\lambda x+(1-\lambda) y, f)|<1$ for all $\left.\lambda \in\right] 0,1[$,

(iii) if $\sup _{f \in X^{\circ}}|(x, f)|=\sup _{f \in X^{\circ}}|(y, f)|=1$ and $x \neq y$, then $\sup _{f \in X^{\circ}}\left|\left(\frac{1}{2}(x+y), f\right)\right|<1$;

(iv) the function $x \rightarrow \sup _{f \in X^{*}}|(x, f)|^{2}, x \in X$, is strictly convex.

PROOF. Let $X$ be strictly convex and let $x, y \in X \backslash\{0\}$ be such that

$$
\sup _{f \in X^{*}}|(x+y, f)|=\sup _{f \in X^{*}}|(x, f)|+\sup _{f \in X^{*}}|(x, f)| .
$$

By Corollary 4 for every $x \in X$, there exists a 
continuous linear functional $x^{*} \in X^{*}$ such that $\left(x+y, x^{*}\right)=\sup _{x^{*} \in X^{*}}\left|\left(x+y, x^{*}\right)\right|, \sup _{x \in X \subset X^{*}}\left|\left(x^{*}, x\right)\right|=1$. Since

$$
\left(x, x^{*}\right) \leq \sup _{f \in X^{\bullet}}|(x, f)|,\left(y, x^{*}\right) \leq \sup _{f \in X^{\bullet}}|(y, f)|
$$

we must have $\left(x, x^{*}\right)=\sup _{f \in X^{*}}|(x, f)|$ and $\left(y, x^{*}\right)=\sup _{f \in X^{*}}|(y, f)|$, i.e.,

$$
\left(\frac{x}{\sup _{f \in X^{*}}|(x, f)|}, x^{*}\right)=\left(\frac{y}{\sup _{f \in X^{*}}|(y, f)|}, x^{*}\right)=1 .
$$

Because $X$ is strictly convex it follows that

$$
\frac{x}{\sup _{f \in X^{\bullet}}|(x, f)|}=\frac{y}{\sup _{f \in X^{\bullet}}|(y, f)|},
$$

hence property (i) holds with

$$
t=\frac{\sup _{f \in X^{*}}|(y, f)|}{\sup _{f \in X^{*}}|(x, f)|} .
$$

To prove that (i) $\rightarrow$ (ii) we assume by contradiction that there exists $x \neq y$ such that $\sup _{f \in X^{*}}|(x, f)|=\sup _{f \in X^{\circ}}|(y, f)|=1$ and $\sup _{f \in X^{*}}|(\lambda x+(1-\lambda) y, f)|=1$, where $\left.\lambda \in\right] 0,1[$. Therefore we have

$$
\sup _{f \in X^{\bullet}}|(\lambda x+(1-\lambda) y, f)|=\sup _{f \in X^{\bullet}}|(\lambda x, f)|+\sup _{f \in X^{*}}|((1-\lambda) y, f)| .
$$

According to property (i) there exists $t \geq 0$ such that $\lambda x=t(1-\lambda) y$. Since $\sup _{f \in X^{\bullet}}|(x, f)|=\sup _{f \in X^{\bullet}}|(y, f)|$ we obtain $\lambda=t(1-\lambda)$ and so $x=y$ which is a contradiction. The implications (ii) $\rightarrow$ (iii) and (iv) $\rightarrow$ (ii) are obvious.

Now we assume that $X$ is not strictly convex Therefore there exist $x_{0}^{*} \in X^{*}$ and $x_{1}, x_{2} \in X$ with $\sup _{x \in X \subset X^{-*}}\left|\left(x^{*}, x\right)\right|=1, \sup _{f \in X^{*}}\left|\left(x_{1}, f\right)\right|=\sup _{f \in X^{*}}\left|\left(x_{2}, f\right)\right|=1, x_{1} \neq x_{2}$ such that $\left(x_{1}, x_{0}\right)=\left(x_{2}, x_{0}^{*}\right)=1$, hence $\left(\frac{1}{2}\left(x_{1}+x_{2}\right), x_{0}^{*}\right)=1$. Thus

$$
\begin{aligned}
\sup _{f \in X^{*}}\left|\left(\frac{1}{2}\left(x_{1}+x_{2}\right), f\right)\right| & =\sup _{\substack{\sup \left|\left(x^{*}, x\right)\right|=1 \\
x \in X \subset X^{* *}}}\left(\frac{1}{2}\left(x_{1}+x_{2}\right), \dot{x}^{*}\right) \\
& \geq\left(\frac{1}{2}\left(x_{1}+x_{2}\right), x_{0}^{*}\right)=1
\end{aligned}
$$

contradicting property (iii). Hence property (iii) implies the strict convexity of $X$. Now, from the equality

$$
\begin{aligned}
\lambda \sup _{f \in X^{*}}|(x, f)|^{2}+(1-\lambda) \sup _{f \in X^{*}}|(y, f)|^{2}= & \left\{\lambda \sup _{f \in X^{*}}|(x, f)|+(1-\lambda) \sup _{f \in X^{*}}|(y, f)|\right\}^{2} \\
& +\lambda(1-\lambda)\left(\sup _{f \in X^{*}}|(x, f)|-\sup _{f \in X^{*}}|(y, f)|\right)^{2}
\end{aligned}
$$

it follows that 


$$
\begin{aligned}
\sup _{f \in X^{*}}|(\lambda x+(1-\lambda) y, f)|^{2} & \leq\left\{\lambda \sup _{f \in X^{\bullet}}|(x, f)|+(1-\lambda) \sup _{f \in X^{\bullet}}|(y, f)|\right\}^{2} \\
& <\lambda \sup _{f \in X^{\bullet}}|(x, f)|^{2}+(1-\lambda) \sup _{f \in X^{\bullet}}|(y, f)|^{2}
\end{aligned}
$$

for all $x, y \in X$ with $\sup _{f \in X^{\bullet}}|(x, f)| \neq \sup _{f \in X^{\bullet}}|(y, f)|$ and $\left.\lambda \in\right] 0,1\left[\right.$. If $\sup _{f \in X^{\bullet}}|(x, f)|=\sup _{f \in X^{\bullet}}|(y, f)|$ we obtain the strict convexity of the function $x \rightarrow \sup _{f \in X^{*}}|(x, f)|^{2}, x \in X$, from (ii). Thus the implication (ii) $\rightarrow$ (iv) is established and the proof is complete.

THEOREM 8. If $X$ is locally convex linear topological space which is strictly convex, then each element $x \in X$ possesses at most one best approximation with respect to a convex set $E \subset X$.

PROOF. Assume by contradiction that there exist two distinct best approximations $l_{1}, l_{2}$ in $E$. Since the set of best approximations is convex, it follows that $\frac{1}{2}\left(l_{1}+l_{2}\right)$ is also a best approximation.

Hence if $d=\inf _{m \in E}\left\{\sup _{f \in X^{*}}|(m-x, f)|\right\}$, we have

$$
\begin{aligned}
0<d=\sup _{f \in X^{*}}\left|\left(x-l_{1}, f\right)\right| & =\sup _{f \in X^{\circ}}\left|\left(x-l_{2}, f\right)\right| \\
& =\sup _{f \in X^{*}}\left|\left(x-\frac{1}{2}\left(l_{1}+l_{2}\right), f\right)\right|
\end{aligned}
$$

where $X^{*}$ is the conjugate space of $X$ and thereby

$$
\sup _{f \in X^{\bullet}}\left|\left(\frac{1}{d}\left(x-l_{1}\right), f\right)\right|=\sup _{f \in X^{\bullet}}\left|\left(\frac{1}{d}\left(x-l_{2}\right), f\right)\right|=1 .
$$

In view of the strict convexity (see Theorem 7) we have

$$
1>\sup _{f \in X^{\cdot}}\left|\left(\frac{1}{2 d}\left(x-l_{1}\right)+\frac{1}{2 d}\left(x-l_{2}\right), f\right)\right|=\frac{1}{d} \sup _{f \in X^{*}}\left|\left(x-\frac{1}{2}\left(l_{1}+l_{2}\right), f\right)\right|=1
$$

which is a contradiction. This completes the proof.

REMARK 2. This property is characteristic of the strictly convex spaces: if, in a locally convex linear topological space $X$, every element possesses at most a best approximation with respect to every convex set (it is enough for the segments), then $X$ is strictly convex.

Indeed, if we assume that $X$ is not strictly convex, then there exists $x, y \in X, x \neq y$, with $\sup _{f \in X^{*}}|(x, f)|=\sup _{f \in X^{*}}|(y, f)|=\sup _{f \in X^{*}}\left|\left(\frac{1}{2}(x+y), f\right)\right|=1$. Furthermore, $\sup _{f \in X^{*}}|(\alpha x+(1-\alpha) y, f)|=1, \forall \alpha \in[0,1]$. Hence the origin has at the best approximation with respect to the closed convex set $[x, y]$ every element of this set, and this clearly contradicts the uniqueness.

From Corollary 2 and Theorem 3 it follows that in a semireflexive strictly locally convex linear topological space, for every closed convex set $E$ we can define the function $P_{E} X \rightarrow X$ by $P_{E} x=l$, where $l$ is the best approximation of $X$ by elements of $E$. This function is called the projection function of the space $X$ into $E$. We note that $P_{E} x \in E$ for every $x \in X$.

DEFINITION 4. Let us consider the following general family of minimization problems

$$
\left(P_{y}\right) \min \{F(x, y) ; x \in X\}, \quad y \in Y
$$

where $X, Y$ are locally convex linear topological spaces and $F: X \times Y \rightarrow \bar{R}$. Let us denote by

$$
h(y)=\inf \{F(x, y) ; x \in X\}, \quad y \in Y
$$

and $H=\{(y, a) \in Y \times R$; there exists $\bar{x} \in X$ such that $F(\bar{x}, y) \leq a\}$. 
THEOREM 9. Let $X, Y$ are locally convex linear topological spaces and $F: X \times Y \rightarrow]-\infty,+\infty]$ be a positively homogeneous and lower-semicontinuous function satisfying the following coercivity condition

$$
F(x, 0)>0 \text { for any } x \in X \mid\{0\} .
$$

Then, if epi $F[1]$ is locally compact, every problem $P_{y}$ has an optimal solution whenever its value is finite.

PROOF. It is easy to observe that

$$
H=\operatorname{Proj}_{Y \times R}(\text { epi } F) .
$$

By hypothesis epi $h$ is a closed cone and so (epi $F)_{\infty}=\operatorname{epi} F$. Therefore, it is sufficient to use Corollary 1.13 ([1], p. 28) for $T=\operatorname{Proj}_{Y \times R}$ and $A=$ epi $F$, taking into account that the separation condition (1.42) of Corollary 1.13 ([1], p. 28) may be written as condition (2.10).

THEOREM 10. If $E$ is a closed locally compact convex set of a strictly convex locally convex linear topological space $X$, then the projection function is continuous on $X$.

PROOF. If $x_{n} \rightarrow x$, for every $\epsilon>0$, then there exists $n_{0}(\epsilon) \in N$ such that $\sup _{f \in X^{*}}\left|\left(x_{n}-x, f\right)\right|<\epsilon$ for all $n>n_{0}(\epsilon)$. Denote

$$
d_{n}=\inf _{m \in E}\left\{\sup _{f \in X^{\bullet}}\left|\left(x_{n}-m, f\right)\right|\right\}, \quad d=\inf _{m \in E}\left\{\sup _{f \in X^{\bullet}}|(x-m, f)|\right\},
$$

where $X^{*}$ is the dual space of $X$.

We have

$$
d_{n} \leq \inf _{m \in E}\left\{\sup _{f \in X^{*}}|(x-m, f)|+\sup _{f \in X^{\bullet}}\left|\left(x_{n}-x, f\right)\right|\right\}<d+\epsilon, \quad \forall n>n_{0}(\epsilon)
$$

hence

$$
\sup _{f \in X^{\bullet}}\left|\left(x-P_{E} x_{n}, f\right)\right| \leq \sup _{f \in X^{\bullet}}\left|\left(x_{n}-P_{E} x_{n}, f\right)\right|+\sup _{f \in X^{*}}\left|\left(x_{n}-x, f\right)\right|<d_{n}+\epsilon<d+2 \epsilon .
$$

Since the set $E \cap \bar{S}(x ; d+\epsilon)$ does not contain any half-line it follows that it is compact where

$$
\bar{S}(x ; d+\epsilon)=\left\{y \in X ; \sup _{f \in X^{\bullet}}|(y-x, f)| \leq d+\epsilon\right\}, \epsilon>0 .
$$

Thus $\bigcap_{\epsilon>0} \bar{S}(x ; d+\epsilon) \cap E \neq \emptyset$ and any subsequence of $P_{E} x_{n}$ has a cluster point $l$ which satisfies $\sup _{f \in X^{\circ}}|(x-l, f)|=d$. Because $X$ is strictly convex, this point is unique and so $P_{E} x_{n} \rightarrow 1=P_{E} x$ as claimed.

DEFINITION 5. A set $E$ is called proximinal if every element of $X$ has a best approximation in $E$. That is, the set $E$ is proximal if the problem

$$
\min _{m \in E}\left\{\sup _{f \in X^{\bullet}}|(x-m, f)|\right\}
$$

has a solution for every $x \in X$.

THEOREM 11. A nonempty set $E$ of a locally convex linear topological space $X$ is proximinal if and only if epi $\sup _{f \in X^{\circ}}|(., f)|+E \times\{0\}$ is closed in $X \times R$.

Moreover, if $E$ is a convex set which contains the origin we have 


$$
\min \left\{\sup _{f \in X^{*}}|(x-m, f)| ; m \in E\right\}=\max \left\{\left(x^{*}, x\right)-P_{E^{0}}\left(x^{*}\right) ; x^{*} \in S^{*} \cap E^{0}\right\}
$$

for every $x \in X$, where $E^{0}$ is the polar set of $E$ ([4], p. 136).

PROOF. Taking in Theorem 2.11 (Chap. $3[1]), f=I_{E}, g=-\sup _{f \in X^{\bullet}}|(., f)|, A=I$, we observe that

$$
\begin{aligned}
& H=\left\{\left(m+x, \sup _{f \in X^{*}}|(x, f)|+r\right) \in X \times R ; m \in E, x \in X, r \geq 0\right\} \\
& =\operatorname{epi} \sup _{f \in X^{\bullet}}|(., f)|+E \times\{0\}
\end{aligned}
$$

as claimed.

REMARK 3. It is easy to see that epi $\sup _{f \in X^{\circ}}|(., f)|=\operatorname{cone}(\bar{S}(0,1) \times\{1\})$, and so if $E$ is a cone, then epi $\sup _{f \in X^{*}}|(., f)|+E \times\{0\}$ is closed in $X \times R$ if and only if $S(0 ; 1)+E$ is closed in $X$. In particular, if $E$ is a linear subspace, denoting by $\phi_{E}: X \rightarrow X / E$ the canonical mapping, the above condition says that $\phi_{E}(\bar{S}(0,1))$ is closed in quotient space $X / E$.

ACKNOWLEDGMENT. The author is most grateful to Dr. R. N. Mukherjee of the Department of Mathematics, University of Burdwan, Burdwan, W.B., India, for his generous encouragement and suggestions at various steps during prepararation of this paper.

\section{REFERENCES}

[1] BARBU, V. and PRECUPANU, TH., Convexity and Optimization in Banach Spaces, D. Reidel Publishing Company (East European Series), 1986.

[2] SINGER, I., Best Approximation in Normed Linear Spaces by Elements of Linear Subspaces, Springer Verlag, Berlin, 1971.

[3] SINGER, I., Generalizations of methods of best approximation to convex optimization in locally convex spaces, I. Rev. Roum. Math. Pures. Appl., 19 (1974), 65-77.

[4] YOSIDA, K., Functional Analysis, Springer Verlag, Berlin, 1965.

[5] KELLEY, J.L. and NAMIOKA, I. and co-authors, Linear Topological Spaces, Springer Verlag, Berlin, 1963. 


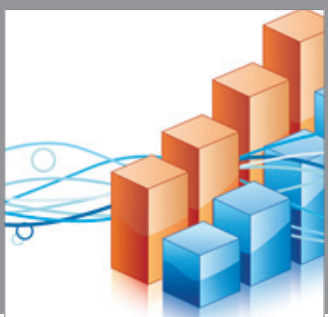

Advances in

Operations Research

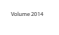

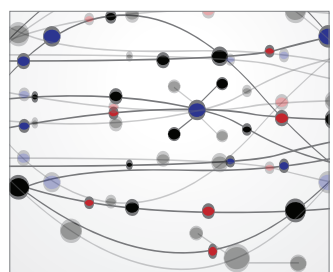

\section{The Scientific} World Journal
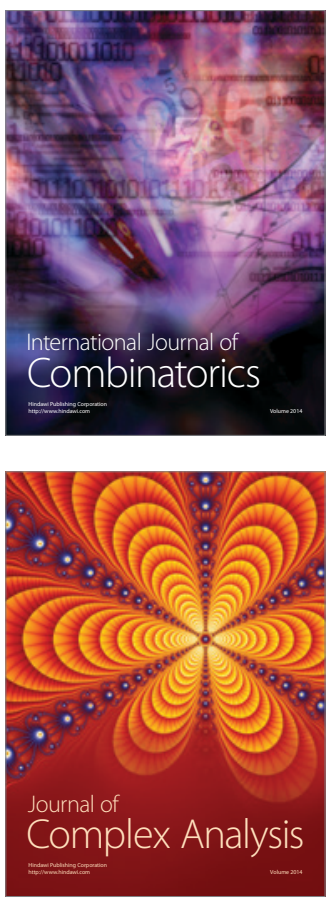

International Journal of

Mathematics and

Mathematical

Sciences
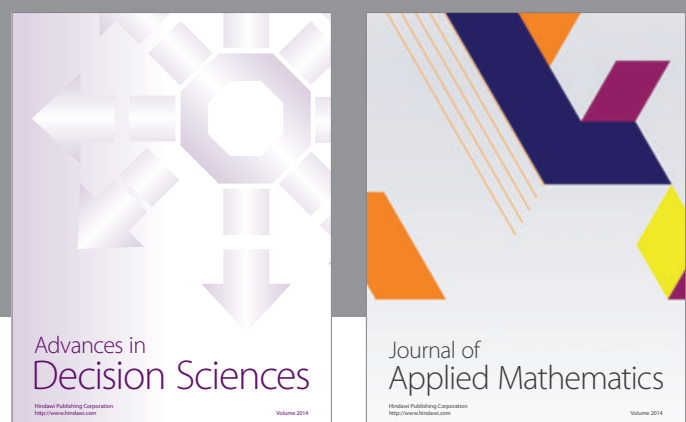

Journal of

Applied Mathematics
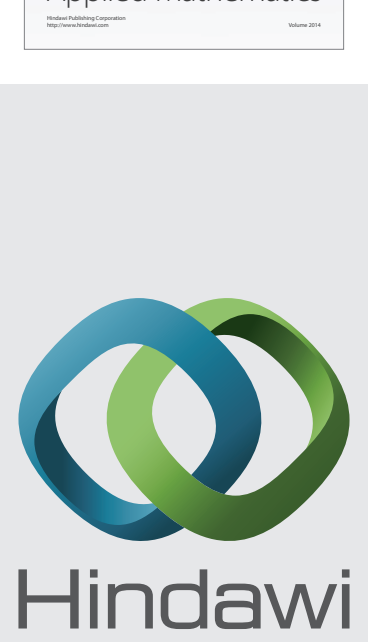

Submit your manuscripts at http://www.hindawi.com
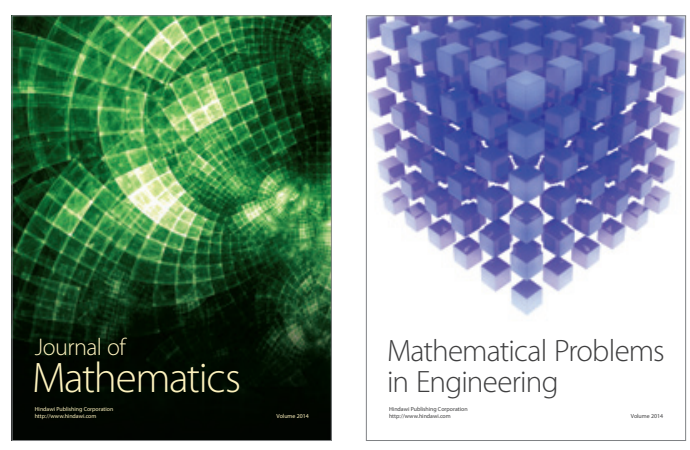

Mathematical Problems in Engineering
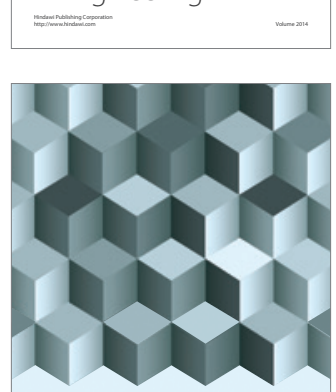

Journal of

Function Spaces
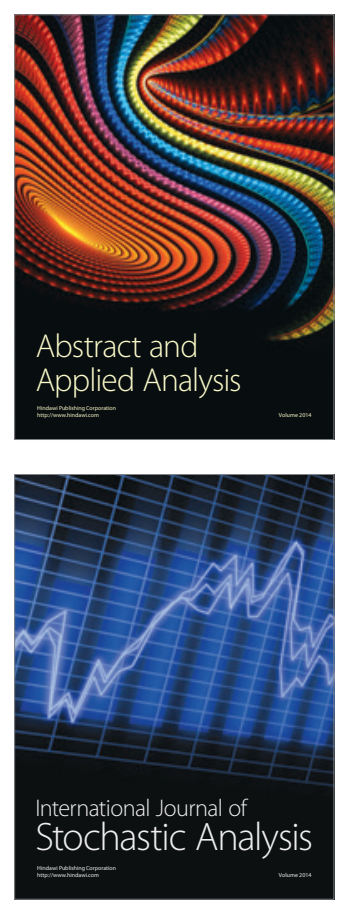

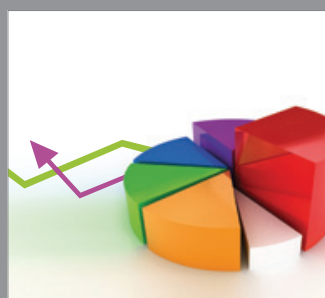

ournal of

Probability and Statistics

Promensencen
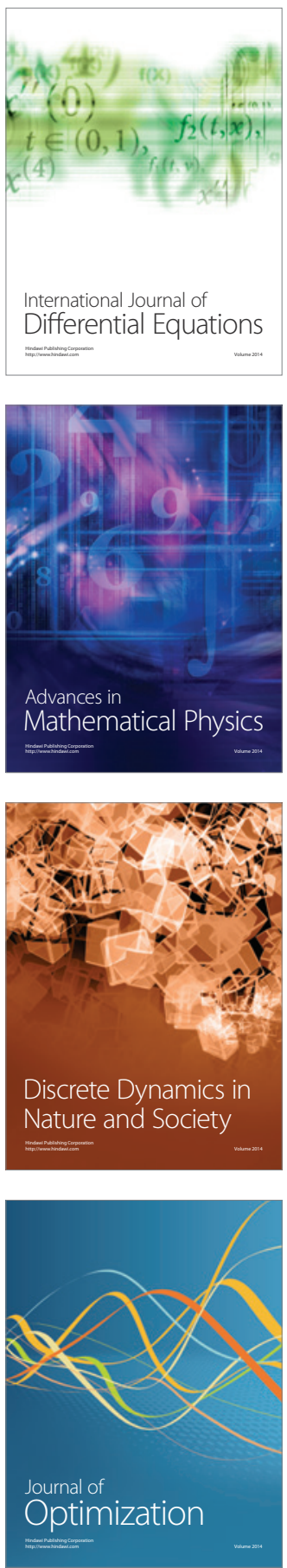